\title{
Evidence for Conical Intersection Dynamics Mediating Ultrafast Singlet Exciton Fission
}

Andrew J. Musser ${ }^{1 *}$, Matz Liebel ${ }^{2 *}$, Christoph Schnedermann ${ }^{2}$, Torsten Wende ${ }^{2}$, Tom B. Kehoe $^{1}$, Akshay Rao ${ }^{1}$, Philipp Kukura ${ }^{2}$

1 Cavendish Laboratory, University of Cambridge

J J Thomson Avenue, Cambridge, UK

2 Physical and Theoretical Chemistry Laboratory, University of Oxford

South Parks Road, Oxford, UK

Email: ar525@cam.ac.uk, philipp.kukura@chem.ox.ac.uk

*These authors contributed equally to this work.

\section{Introductory Paragraph}

Singlet exciton fission is the process in organic semiconductors through which a spin-singlet exciton converts into a pair of spin-triplet excitons residing on different chromophores, entangled in an overall spin-zero state. For some systems, singlet fission has been shown to occur on the 100 fs timescale and with a $200 \%$ yield, but the mechanism of this process remains uncertain. Here we study a model singlet fission system, TIPS-pentacene, using ultrafast vibronic spectroscopy. We observe that vibrational coherence in the initially photogenerated singlet state is transferred to the triplet state and show that this behaviour is effectively identical to that observed in ultrafast internal conversion for polyenes in solution. This similarity in vibronic dynamics suggest that both multi-molecular singlet fission and single-molecular internal conversion are mediated by the same underlying relaxation processes, based on strong coupling between nuclear and electronic degrees of freedom. In its most efficient form this leads to a conical intersection between the coupled electronic states. 


\section{Main Text}

Singlet exciton fission has long commanded interest as an exceptionally fast channel to generate triplet excitons in organic materials. At the heart of the process is the coupling of the final triplet pair to the initially photoexcited singlet state, which ensures conservation of $\operatorname{spin}^{1}$. In systems where singlet fission is exergonic, it can be rapid and highly efficient. For instance, thin films of pentacene and TIPS-pentacene exhibit triplet formation with a time constant of $80 \mathrm{fs}$ and yields of $200 \% \%^{2,3}$. Current interest in this phenomenon is driven by its potential to circumvent the Shockley-Queisser limit for single-junction solar cells. By converting high-energy photons into two low-energy excited states, singlet fission offers a means to overcome thermalisation losses. Devices based on pentacene, a fission sensitiser, have demonstrated external quantum efficiencies of $129 \%$, the highest for any photovoltaic technology to date ${ }^{4}$.

Current theoretical descriptions of singlet fission are framed by the kinetic model proposed by Johnson and Merrifield in $1970^{5}$, which established the role of a triplet pair (TT) coupled into an overall singlet state as an intermediate to triplet formation, but the details of the ultrafast fission process are subject to debate. Most theoretical studies of the canonical system, pentacene, focus on the low-lying electronic states and their composition by TT, intermolecular charge-transfer and monomolecular singlet configurations ${ }^{6-9}$. The relative strengths of the direct electronic couplings between these states then determine the overall fission mechanism, proposals for which can be roughly divided into two classes. In the 'direct' model, the TT state is formed by electronic coupling between the initial singlet and the TT manifolds, and singlet-to-triplet conversion is accomplished through an avoided crossing or a conical intersection ${ }^{9-11}$. Alternatively, the 'mediated' model proposes that the initial singlet couples much more strongly to virtual charge-transfer configurations ${ }^{6,7}$ and, in some descriptions, may even directly populate charge-transfer states ${ }^{1,8}$. These charge-transfer configurations, whether real or virtual, in turn have strong coupling to TT and enable singlet fission. The limit of very strong coupling between singlet and this intermediate state results in the 'coherent' model, where the photoexcited singlet state and TT are linked by electronic coherence $^{12}$. The recent study by Yost et al. ${ }^{3}$ suggests that two of these mechanisms, direct and virtual mediated coupling, may contribute to determining the rate of singlet fission in the acenes, with the weight of mediated coupling dependent on the contribution of chargetransfer character to the first excited state. 
In contrast to the rigorous theoretical debate, there is little experimental data available that helps our understanding of the mechanism behind singlet fission. Standard experimental techniques such as transient absorption or photoluminescence spectroscopy enable direct monitoring of singlet and triplet exciton populations and the kinetics of their interconversion $^{2,13-17}$. These methods, however, are generally insensitive to the nature of the coupling between states. More sophisticated spectroscopic approaches are therefore required to build a clear picture of such underlying dynamics.

At the same time, there is a growing awareness of the central role of vibronic coupling in a range of ultrafast photophysical processes, from photosynthesis to charge separation in organic heterojunctions ${ }^{18-21}$. Many ultrafast (<200 fs) photophysical processes, such as internal conversion in isolated molecules, have been studied experimentally and theoretically in detail and are generally accepted to involve conical intersections between the electronic states induced by strong coupling between vibrational and electronic degrees of freedom ${ }^{22,23}$. The ultrafast time scales for fission point to the importance of understanding the associated nuclear dynamics that mediate the conversion of singlets to triplets, but no experimental information on the underlying vibronic coupling is currently available. To probe the involved nuclear dynamics and thereby provide new insight on the mechanism of ultrafast singlet fission, we approach the problem with a recently developed experimental technique that uses impulsively generated vibrational coherence as a probe of vibronic coupling ${ }^{24}$. We study thin films of TIPS-pentacene (Fig. 1a), a solution-processable pentacene derivative that undergoes efficient fission on sub-100 fs timescales (Fig. 1b), making it a model system for the study of ultrafast singlet fission ${ }^{3}$. Our results reveal that vibrational wavepackets generated in the initially populated first excited singlet state $\left(\mathrm{S}_{1}\right)$ transfer to the TT state through the process of singlet fission. We show that this behaviour is effectively identical to that observed during ultrafast internal conversion for isolated polyene molecules in solution, suggesting that they are subject to the same underlying mechanism.

\section{Ultrafast Vibronic Spectroscopy}

Our experimental technique consists of two time-resolved spectroscopic measurements, performed in parallel on the same sample (Fig. 1c). The 'two-pulse' experiment is equivalent to transient absorption spectroscopy using a temporally compressed, broadband pump pulse. 
In brief, a 10 fs pulse of light, the 'pump', excites the sample, and after a time delay ranging from femtoseconds to picoseconds, a broadband white-light continuum pulse, the 'probe', interrogates the same sample region. We resolve the probe transmission through the film spectrally and temporally and compare its wavelength-dependent intensity with and without the pump pulse. The resulting normalised differential transmittance, $\Delta \mathrm{T} / \mathrm{T}$, reflects the spectral signatures of the transient photogenerated states, where positive features indicate the bleaching of the ground-state absorption or stimulated emission and negative features correspond to characteristic absorption bands of excited electronic states. The use of a temporally compressed pump pulse with a duration shorter than most vibrational periods is essential, as it generates nuclear wavepackets in all Franck-Condon active nuclear degrees of freedom on the ground and photoexcited singlet states ${ }^{24-26}$. The resulting vibrational coherences manifest themselves as oscillations in the transient absorption kinetics and correspond to the Raman active modes of the involved electronic states ${ }^{27}$.

The interpretation of the underlying vibrational signatures in such a two-pulse experiment, however, is not trivial, as they are often contaminated by ground state features. To isolate excited state coherences, we extend the commonly employed 'two-pulse' scheme with a third pulse that modifies the excited state population without interacting with molecules that have remained in the ground electronic state. In this experiment, the ultrashort pump populating the first excited state is immediately followed by a comparatively long (650 fs; equivalent results were achieved with a $250 \mathrm{fs}$ pulse) 'dump' pulse. The dump is solely resonant with the photo-induced absorption band of the excited state of interest, so as to remove a subpopulation of excitons by excitation into a higher-lying electronic state. We tuned the dump pulse to $860 \mathrm{~nm}$, corresponding to the $\mathrm{T}_{1} \rightarrow \mathrm{T}_{2}$ transition of the TIPS-pentacene triplet. In this way, the dump pulse removes population from the lowest lying $\mathrm{T}_{1}$ state by exciting it to a higher lying state, which in turn removes the vibrational coherence of this subset of triplet excitons. A comparison of 'dump on' and 'dump off' configurations then isolates residual vibrational coherence signatures of the triplet state.

\section{Ground-State Vibrational Coherence}

The transient absorption data from the two-pulse experiment are presented in Fig. 2a. We observe a rapid decay of the TIPS-pentacene stimulated emission at $700 \mathrm{~nm}$ with simultaneous formation of the triplet excited state absorption bands below $550 \mathrm{~nm}$ and above $750 \mathrm{~nm}$, with an exponential time constant of $80 \mathrm{fs}$, consistent with previous reports (see 
Supplementary Fig. S1) ${ }^{3}$. Once formed, the triplet population remains stable throughout the 2200 fs detection window. On the right we present the transient spectrum integrated from 1000 to 2200 fs. In addition to the two well-known triplet absorption bands below $560 \mathrm{~nm}$ and above $730 \mathrm{~nm}^{13,14}$, we detect a series of small positive peaks. These precisely match the shape of the ground-state absorption spectrum of the film (grey), confirming their assignment to bleaching of the ground-state absorption. Figure $2 \mathrm{~b}$ contains a zoom of the same data at early time delays, allowing better resolution of the triplet formation process, particularly in the background-free growth of the $T_{1} \rightarrow T_{2}$ absorption band. Here we can directly resolve the effect of the temporally compressed pump pulse, leading to oscillations along every contour line. These weak modulations are not noise, but rather a direct consequence of the short pump pulse launching nuclear wavepackets on ground and excited electronic states.

To reveal the molecular origin of the observed coherent oscillations, we subtract the slowly varying electronic population kinetics and retrieve the pure vibrational coherence (Supplementary Fig. S2). We can then construct the spectrally resolved frequency content through a Fourier transform of the oscillatory kinetics at each probe wavelength (Fig. 3a). Well-defined vibrational frequencies are observed across the entire spectral range. Integration in the region of ground-state absorption, such as the GSB band indicated in the FFT map, reproduces the expected spontaneous resonance Raman spectrum (light and dark grey, Figure $3 b)$, i.e. the time-domain oscillations highlighted in Fig. $2 b$ follow the normal vibrational modes of the molecule in its ground electronic state. Our technique can thus be regarded as the time-domain equivalent of resonance Raman spectroscopy ${ }^{28}$. The frequencies are insensitive to the selection of the integration band, but the regions indicated were chosen to minimise contamination from overlapping ground-state bleach and excited-state absorption features around $600 \mathrm{~nm}$ and $775 \mathrm{~nm}$. For instance, the frequency spectrum in the $\mathrm{T}_{1} \rightarrow \mathrm{T}_{3}$ absorption band (light blue) exhibits the same ground-state vibrational modes as well as strong enhancement of several modes not found in the spontaneous Raman spectrum, such as the bands at $1204 \mathrm{~cm}^{-1}$ and $1407 \mathrm{~cm}^{-1}$. This variation in relative FFT intensities between the triplet absorption bands and the central ground-state bleach region indicates spectral selectivity suggestive of excited-state vibrational coherence, but in the two-pulse experiment alone they cannot be conclusively separated.

\section{Excited State Vibrational Coherence}


Using electronic population control in the three-pulse experiment, we can remove residual ground-state signatures and isolate vibrational coherence on the final excited state. The dump pulse at $860 \mathrm{~nm}$ acts as a small perturbation to the system (Supplementary Figs. S3 and S4) and does not disturb the singlet fission process. Importantly, the application of an additional, long dump pulse only changes the excited state population and the vibrational coherence of those molecules it directly excites. It has a negligible effect on the vibrational coherence of the molecules with which it is not resonant ${ }^{26}$. The resulting vibrational coherence map in Fig. $3 \mathrm{c}$ shows complete removal of coherence activity in the central, ground state bleach region and of all ground-state vibrational modes across the spectrum. The comparison between the two Fourier maps (Fig. 3a and 3c) demonstrates that the dump pulse does not perturb the ground state population, resulting in an optimal subtraction of any remaining ground state features. As a result, the only residual coherent activity appears in the spectral regions associated with the triplet absorption bands $\mathrm{T}_{1} \rightarrow \mathrm{T}_{3}$ and $\mathrm{T}_{1} \rightarrow \mathrm{T}_{2}$.

We emphasise the clear difference between the ground state Raman spectrum of TIPSpentacene (light and dark grey) and the isolated excited state coherence (dark blue). Comparison between the spontaneous and time-domain Raman spectra shows similarities with all major bands matching in vibrational frequency. Differences in the relative magnitudes of the bands are caused largely by the finite temporal resolution of the experiment, which leads to an underestimation of the Raman intensities of higher-frequency modes $\left(>1200 \mathrm{~cm}^{-1}\right)$. The isolated excited state coherence, however, reveals a completely altered spectral pattern. All major ground state bands (788, 1158, 1195, 1374, 1508, 1578 and $1626 \mathrm{~cm}^{-1}$ ) are no longer present, and instead significantly shifted or completely new bands (799, 1206, 1342, 1409 and $1567 \mathrm{~cm}^{-1}$ ) are observed. A comparison of the excited state coherence (Fig. 3c, dark blue) with vibrational coherence directly generated in the triplet state in a separate experiment (Supplementary Figs. S5-6), together with a spectral intensity dependence following the triplet absorption spectra, confirms the assignment to coherent oscillations within the triplet manifold.

\section{Vibronic Coupling}

We recall from the presentation of the electronic population kinetics in Fig. 2 that the triplet population is not directly excited, but rather grows in with an $80 \mathrm{fs}$ time constant. It follows that the vibrational coherence we observe cannot be directly produced in this state by the pump pulse. Nor can this coherence be produced spontaneously through singlet fission, along 
the lines of the impulsive mechanism proposed by Burdett et al. ${ }^{15}$ to explain much slower oscillations observed in tetracene delayed fluorescence. While rapid electronic population transfer can often be treated as an effective laser pulse, the oscillations we detect here exhibit periods which are significantly faster than the 80 fs triplet formation time: we observe frequencies of up to $1567 \mathrm{~cm}^{-1}$, corresponding to a period of just $21 \mathrm{fs}$. It follows that the vibrational coherence must have been produced by the compressed pump pulse and subsequently transferred from the photoexcited singlet state to the triplets through the process of singlet exciton fission.

Our observation of efficient coherence transfer is reminiscent of theoretical predictions for the fates of nuclear wavepackets undergoing internal conversion between electronic states ${ }^{23}$. To allow for an experimental comparison with a well-understood molecular system exhibiting a simple electronic structure, we have performed the same two-pulse and threepulse experiments on dilute solutions of diphenyloctatetraene (DPO). Due to symmetry restrictions, photoexcitation of this polyene only populates the $1 \mathrm{~B}_{\mathrm{u}}$ state, which exhibits a characteristic photoinduced absorption in the NIR. This state is not the lowest in energy, and rapidly (70 fs) converts into the one-photon-forbidden $2 \mathrm{~A}_{\mathrm{g}}$ state via a conical intersection with unity efficiency (Fig. 4a,b). As described above, excitation with a temporally compressed pulse (14 fs) directly produces vibrational coherence within the initial $1 \mathrm{~B}_{\mathrm{u}}$ excited state. Nevertheless, the three-pulse experiment reveals significant vibrational coherence in the $2 \mathrm{~A}_{\mathrm{g}}$ state, as shown in Figure 4c; passage through the conical intersection results in transfer of vibrational coherence between electronic states. We stress the strong similarity of the vibronic dynamics observed in DPO - a simple small-molecule system with only two low-lying excited electronic states, linked by a conical intersection - and thin films of TIPS-pentacene. We thus propose that singlet fission in TIPS-pentacene is mediated by a conical intersection between the singlet and triplet-pair manifolds, as described schematically in Figure 5, similar to the conical intersection between the $1 \mathrm{~B}_{\mathrm{u}}$ and $2 \mathrm{~A}_{\mathrm{g}}$ states in DPO. Despite this remarkable similarity, however, we highlight a critical distinction. Whereas all the excited states of DPO reside on the same molecule, in the case of singlet fission the TT state resides on two molecules. This implies that more than one molecule is involved in the conical intersection between singlet and TT manifolds.

\section{Discussion}


These results further point to the importance of exciton delocalisation for ultrafast triplet formation. Triplet generation through singlet exciton fission is mediated by the formation of the intrinsically intermolecular triplet-pair state TT, involving a pair of individually localised triplet excitons which is spread over a minimum of two molecules ${ }^{9}$. Likewise, then, the vibrational coherence shown in Fig. 3c must have passed through the intermolecular TT state and reflects a coherent superposition of localised molecule modes. Motion of the wavepacket along the TT potential energy surface entails vibrations of all molecules contributing to the excited state. We know of no mechanism for the vibrational coherence to be spontaneously generated in an initially stationary molecule by spreading of the TT wavefunction alone and consider it more probable that the coherent oscillations were present in all participating molecules from the initial photoexcitation. In other words, the singlet exciton and its attendant vibrational coherence must have been delocalised prior to singlet fission. Though singlet fission can still proceed efficiently from fully localised states in solution ${ }^{14}$, we propose that the ultrafast fission regime discussed in this work requires an initial state that is delocalised over more than one molecule.

Our experimental evidence for ultrafast singlet fission mediated by a conical intersection agrees with the predictions, based on ab initio calculations, of Zimmerman et al. ${ }^{10}$. While our results are consistent with this 'direct' model of singlet fission, we do not consider the agreement to be exclusive. Later ab initio studies by Feng et al. ${ }^{29}$ and Casanova $^{30}$ have similarly implicated vibronic coupling between the singlet and triplet-pair manifolds while also highlighting the role of charge-transfer contributions to both of these states. It is thus possible that a superexchange-type 'mediated' model could also account for ultrafast singlet fission via a concial intersection given sufficiently strong charge transfer character in the photoexcited singlet state $^{3}$, though such models will need to be reformulated beyond the standard diabatic treatment ${ }^{7}$. Mechanisms involving sequential physical charge transfer can, however, be ruled out: there is no precedent for such a typically stochastic process to transfer vibrational coherence. There is a priori no reason why nuclear motion in the product state formed by a purely electronic interaction should be correlated to the originally populated state if they are not vibronically coupled. The well-resolved formation of triplet excitons from zero baseline is also inconsistent with models involving strong electronic coherence ${ }^{12}$, where a superposition of singlets and triplet pairs is generated directly via photoexcitation; the triplets are rather generated from the photoexcited singlet population over 80 fs. 


\section{Conclusions}

In conclusion, we have experimentally elucidated the role of vibrational motion in ultrafast singlet exciton fission. We demonstrated that the correlated triplet pair generated via fission and the initially populated Franck-Condon region in the singlet state are fundamentally connected as would be expected for a single, multi-dimensional potential energy surface. Following photoexcitation, particular 'driving' modes efficiently shuttle the singlet exciton wavepacket into the region of the state crossing, where the singlet and TT potential energy surfaces are linked through vibronic coupling, giving rise to signatures of a multi-molecular conical intersection. This simple picture explains the high rate and efficiency of singlet fission in TIPS-pentacene and is consistent with established photophysics of isolated polyenes such as DPO. Our results indicate that a complete theoretical description of singlet fission in the ultrafast regime will need to explicitly consider the effects of nuclear motion and exciton delocalisation. Irrespective of the model chosen, however, we emphasise that our data demonstrate that the vibronic signatures of ultrafast singlet excition fission involving two molecules are indistinguishable from those for an ultrafast internal conversion in a single molecule. Although, in principle, complex mechanisms can be invoked to explain the fission process, our data suggests fission and internal conversion are in fact remarkably similar and based in a simple but efficient breakdown of the Born-Oppenheimer approximation resulting in a strong coupling of nuclear and electronic degrees of freedom.

Given the prevalence of conical intersections in fast photophysical processes, we anticipate that the same model can be generalised to other ultrafast singlet fission systems such as pentacene $^{2}$ and the polyenes ${ }^{31,32}$. Indeed, the widely accepted presence of conical intersections in polyenes offers the possibility that this mechanism may span the two major classes of singlet fission chromophores ${ }^{1}$. A combination of the techniques used in this study with higher-order measurements such as $2 \mathrm{D}$ electronic spectroscopy ${ }^{33}$ should be able to identify the nuclear modes that drive the system towards the crossing and that modulate the strength of the vibronic coupling between singlet and TT, which may enable more effective rational design of new singlet fission sensitisers. Such studies may additionally shed new light on the behaviour and properties of conical intersections and could provide a potent tool to explore how they are affected by changes in crystal packing, intermolecular coupling and electronic structure. 


\section{References:}

1. Smith, M. B. \& Michl, J. Singlet Fission. Chem. Rev. 110, 6891-6936 (2010).

2. Wilson, M. W. B. et al. Ultrafast dynamics of exciton fission in polycrystalline pentacene. J. Am. Chem. Soc. 133, 11830-11833 (2011).

3. Yost, S. R. et al. A transferable model for singlet-fission kinetics. Nat. Chem. 6, 492497 (2014).

4. Congreve, D. N. et al. External quantum efficiency above $100 \%$ in a singlet-excitonfission-based organic photovoltaic cell. Science 340, 334-337 (2013).

5. Johnson, R. C. \& Merrifield, R. E. Effects of Magnetic Fields on the Mutual Annihiliation of Triplet Excitons in Anthracene Crystals. Phys. Rev. B 1, 896-902 (1970).

6. Beljonne, D., Yamagata, H., Brédas, J. L., Spano, F. C. \& Olivier, Y. Charge-Transfer Excitations Steer the Davydov Splitting and Mediate Singlet Exciton Fission in Pentacene. Phys. Rev. Lett. 110, 226402 (2013).

7. Berkelbach, T. C., Hybertsen, M. S. \& Reichman, D. R. Microscopic theory of singlet exciton fission. II. Application to pentacene dimers and the role of superexchange. $J$. Chem. Phys. 138, 114103 (2013).

8. Greyson, E. C., Vura-Weis, J., Michl, J. \& Ratner, M. A. Maximizing singlet fission in organic dimers: theoretical investigation of triplet yield in the regime of localized excitation and fast coherent electron transfer. J. Phys. Chem. B 114, 14168-14177 (2010).

9. Zimmerman, P. M., Musgrave, C. B. \& Head-Gordon, M. A Correlated Electron View of Singlet Fission. Acc. Chem. Res. 46, 1339-1347 (2013).

10. Zimmerman, P. M., Zhang, Z. \& Musgrave, C. B. Singlet fission in pentacene through multi-exciton quantum states. Nat. Chem. 2, 648-52 (2010).

11. Renaud, N., Sherratt, P. A. \& Ratner, M. A. Mapping the Relation between Stacking Geometries and Singlet Fission Yield in a Class of Organic Crystals. J. Phys. Chem. Lett. 4, 1065-1069 (2013).

12. Chan, W.-L. et al. The Quantum Coherent Mechanism for Singlet Fission: Experiment and Theory. Acc. Chem. Res. 46, 1321-1329 (2013).

13. Rao, A., Wilson, M. W. B., Albert-Seifried, S., Di Pietro, R. \& Friend, R. H. Photophysics of pentacene thin films: The role of exciton fission and heating effects. Phys. Rev. B 84, 195411 (2011). 
14. Walker, B. J., Musser, A. J., Beljonne, D. \& Friend, R. H. Singlet Exciton Fission in Solution. Nat. Chem. 5, 1019-1024 (2013).

15. Burdett, J. J. \& Bardeen, C. J. Quantum beats in crystalline tetracene delayed fluorescence due to triplet pair coherences produced by direct singlet fission. J. Am. Chem. Soc. 134, 8597-8607 (2012).

16. Burdett, J. J., Piland, G. B. \& Bardeen, C. J. Magnetic field effects and the role of spin states in singlet fission. Chem. Phys. Lett. 585, 1-10 (2013).

17. Wilson, M. W. B. et al. Temperature-Independent Singlet Exciton Fission in Tetracene. J. Am. Chem. Soc. 135, 16680-16688 (2013).

18. Tiwari, V., Peters, W. K. \& Jonas, D. M. Electronic resonance with anticorrelated pigment vibrations drives photosynthetic energy transfer outside the adiabatic framework. Proc. Natl. Acad. Sci. U. S. A. 110, 1203-1208 (2013).

19. Chin, A. W. et al. The role of non-equilibrium vibrational structures in electronic coherence and recoherence in pigment--protein complexes. Nat. Phys. 9, 113-118 (2013).

20. Gélinas, S. et al. Ultrafast Long-Range Charge Separation in Organic Semiconductor Photovoltaic Diodes. Science 343, 512-516 (2014).

21. Halpin, A. et al. Two-dimensional spectroscopy of a molecular dimer unveils the effects of vibronic coupling on exciton coherences. Nat. Chem. 6, 196-201 (2014).

22. Köppel, H., Domcke, W. \& Cederbaum, L. S. in Adv. Chem. Physics, Vol LVII (Prigogine, I. \& Rice, S. A.) LVII, 59-246 (John Wiley \& Sons, 1984).

23. Kühl, A. \& Domcke, W. Multilevel Redfield description of the dissipative dynamics at conical intersections. J. Chem. Phys. 116, 263 (2002).

24. Liebel, M., Schnedermann, C. \& Kukura, P. Vibrationally Coherent Crossing and Coupling of Electronic States during Internal Conversion in $\beta$-Carotene. Phys. Rev. Lett. 112, 198302 (2014).

25. Ruhman, S., Joly, A. G. \& Nelson, K. A. Coherent molecular vibrational motion observed in the time domain through impulsive stimulated Raman scattering. IEEE J. Quantum Electron. 24, 460-469 (1988).

26. Wende, T., Liebel, M., Schnedermann, C., Pethick, R. J. \& Kukura, P. Populationcontrolled impulsive vibrational spectroscopy: background- and baseline-free Raman spectroscopy of excited electronic States. J. Phys. Chem. A 118, 9976-84 (2014).

27. Pollard, W. T. \& Mathies, R. A. Analysis of femtosecond dynamic absorption spectra of nonstationary states. Annu. Rev. Phys. Chem. 43, 497-523 (1992).

28. Johnson, A. E. \& Myers, A. B. A comparison of time- and frequency-domain resonance Raman spectroscopy in triiodide. J. Chem. Phys. 104, 2497 (1996). 
29. Feng, X., Luzanov, A. V. \& Krylov, A. I. Fission of Entangled Spins: An Electronic Structure Perspective. J. Phys. Chem. Lett. 4, 3845-3852 (2013).

30. Casanova, D. Electronic structure study of singlet-fission in tetracene derivatives. $J$. Chem. Theory Comput. 10, (2014).

31. Musser, A. J. et al. Activated Singlet Exciton Fission in a Semiconducting Polymer. $J$. Am. Chem. Soc. 135, 12747-12754 (2013).

32. Wang, C. \& Tauber, M. J. High-yield singlet fission in a zeaxanthin aggregate observed by picosecond resonance Raman spectroscopy. J. Am. Chem. Soc. 132, 13988-13991 (2010).

33. Kitney-Hayes, K. A., Ferro, A. A., Tiwari, V. \& Jonas, D. M. Two-dimensional Fourier transform electronic spectroscopy at a conical intersection. J. Chem. Phys. 140, 124312 (2014).

\section{Acknowledgements}

A.R. thanks Corpus Christi College, Cambridge for a Research Fellowship. T.W. was supported by a Marie Curie Intra European Fellowship [PIEF-GA-2013-623652]. P.K. was supported by the Engineering and Physical Sciences Research Council [EPSRC, EP/H003541]. This work was supported by the EPSRC and the Winton Programme for the Physics of Sustainability.

\section{Author Contributions}

A.J.M. and M.L. analysed the data, M.L., C.S. and T.W. carried out the time-resolved experiments, T.K. performed the steady-state Raman spectroscopy, A.R. and P.K. conceived of the project. All authors discussed the results, A.J.M., A.R. and P.K. wrote the manuscript with input from all authors.

\section{Additional information}

Supplementary information is available in the online version of the paper. Reprints and permissions information is available online at www.nature.com/reprints. Correspondence and requests for materials should be addressed to A.R. and P.K.

\section{Competing financial interests}

The authors declare no competing financial interests. 


\section{Figure Captions}

Figure 1 Photophysics and relevant transitions for impulsive vibrational spectroscopy of TIPS-pentacene. a Chemical structure of TIPS-pentacene and visible absorption spectrum of a thin film. b Low-lying electronic states (black), excited-state optical transitions (red dashed) and key photophysical processes (green) following excitation of TIPS-pentacene. Singlet exciton fission from $S_{1}$ occurs on a sub-100-fs timescale, initially forming a coupled triplet pair, at energy $T_{1}+T_{1}$ (grey dashed). No distinct optical signatures for this state have been identified in the solid state, which eventually dissociates to form free triplet excitons. c Pulse schemes. The two-pulse experiment is equivalent to standard transient absorption, using a compressed broadband excitation pulse (blue) to establish vibrational coherence in the ground and excited states. In the three-pulse scheme an additional temporally stretched dump pulse (purple) arrives at the same time as the pump and is tuned to the photoinduced absorption of the excited state of interest, selectively removing triplet population and thus vibrational coherence on that state.

Figure 2 Transient absorption spectroscopy of TIPS-pentacene excited with a resonant $10 \mathrm{fs}$ pulse. a Differential transmission of TIPS-pentacene as a function of probe wavelength in the two-pulse experiment, showing the full temporal evolution explored in this study. Within 500 fs, the initial dynamics are complete and we observe only triplet excitons. The integrated spectrum (right, solid line) indicates the positions of the $T_{1}$ excited state absorption bands (blue and green) and the ground-state absorption (grey), which aligns with the ground-state bleach (GSB) peaks in the middle of the spectrum. b Early-time dynamics from panel a. The initial stimulated emission at $700 \mathrm{~nm}$ decays to yield triplets with excited state absorption bands at $520 \mathrm{~nm}$ and $820 \mathrm{~nm}$, which grow in with an 80 fs time constant. The compressed pump pulse generates vibrational coherence throughout the spectrum, visible here as oscillations between contours.

Figure 3 Vibrational coherence transfer during singlet exciton fission. a Fourier transform power map of the two-pulse dataset after subtraction of the electronic kinetics, showing strong vibrational coherence at similar frequencies across the spectral window, with particularly large signatures in the GSB region $600-750 \mathrm{~nm}$. b Integration across the GSB band indicated in a (dark grey) reproduces the main frequencies of the spontaneous Raman spectrum (light grey). The redistribution of peak intensities between the GSB region and the triplet band $\mathrm{T}_{1} \rightarrow \mathrm{T}_{3}$ (light blue) indicates a mixture of contributions from ground-state (GS) 
and excited-state (ES) coherence signatures. Integration of the frequency map from the threepulse experiment (dark blue) reveals only the distinct vibrational modes of the triplet exciton, demonstrating that vibrational coherence established under photoexcitation is transferred via the process of singlet fission. Major vibrational bands have been labelled for clarity. See supplementary information for frequency spectra of the other regions indicated. c Subtraction of the ground-state coherence using the three-pulse scheme reveals residual coherence in the region of the triplet excited state absorption bands, while little activity remains in the GSB region. Several ground-state vibrational modes, such as the previously dominant band at $1163 \mathrm{~cm}^{-1}$, are completely removed.

Figure 4 Vibrational coherence transfer in DPO. a Chemical structure and low-lying electronic states of DPO. States populated following photoexcitation at $380 \mathrm{~nm}$ are indicated in black. Direct one-photon excitation into the $2 \mathrm{~A}_{\mathrm{g}}$ electronic state is symmetry-forbidden. Conversion from $1 \mathrm{~B}_{\mathrm{u}}$ to $2 \mathrm{~A}_{\mathrm{g}}$ is monitored through the indicated excited-state optical transitions (red dashed). b Transient absorption map of DPO in a two-pulse experiment. The photoexcited $1 \mathrm{~B}_{\mathrm{u}}$ state undergoes ultrafast internal conversion into the $2 \mathrm{~A}_{\mathrm{g}}$ state via a conical intersection, with a70 fs time constant. Spectra were collected up to 2.5 ps pump-probe delay and revealed no further evolution on this time scale. Spectra (right) at $100 \mathrm{fs}\left(1 \mathrm{~B}_{\mathrm{u}}\right)$ and $500 \mathrm{fs}$ $\left(2 \mathrm{~A}_{\mathrm{g}}\right)$ show the signatures of these two states. c Excited-state vibrational coherence was measured using the three-pulse scheme analogous to TIPS-pentacene. Vibrational coherence in the $2 \mathrm{~A}_{\mathrm{g}}$ state (dark orange) was integrated over the region indicated in $\mathbf{b}$, and is fully distinct in band intensities and frequencies from the spontaneous Raman spectrum (grey).

Figure 5 Schematic of singlet fission via a conical intersection. The wavepacket is driven from the Franck-Condon region to the intersection seam connecting the $S_{1}$ and TT potential energy surfaces. The singlet wavefunction prior to fission (left) is delocalised over at least two molecules, and following passage through the conical intersection the triplet pair wavefunction is also delocalised (right). The triplet pair state will eventually localise to two isolated triplets on two molecules. 
Figure 1

a

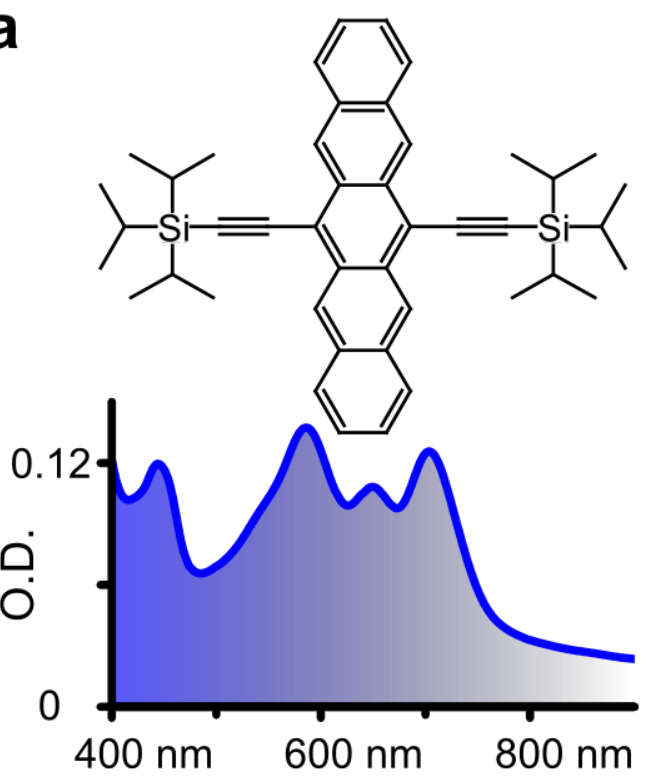

b

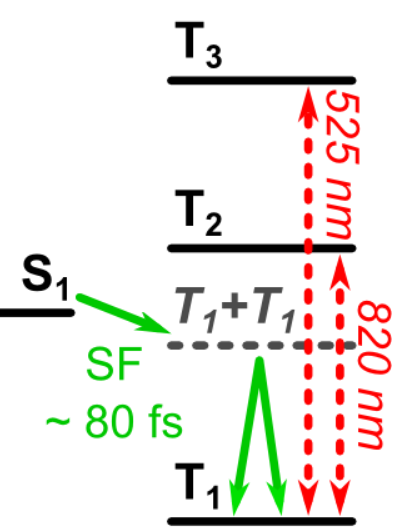

C

Two-Pulse

Experiment

Three-Pulse

Pump Probe Pump Dump Probe
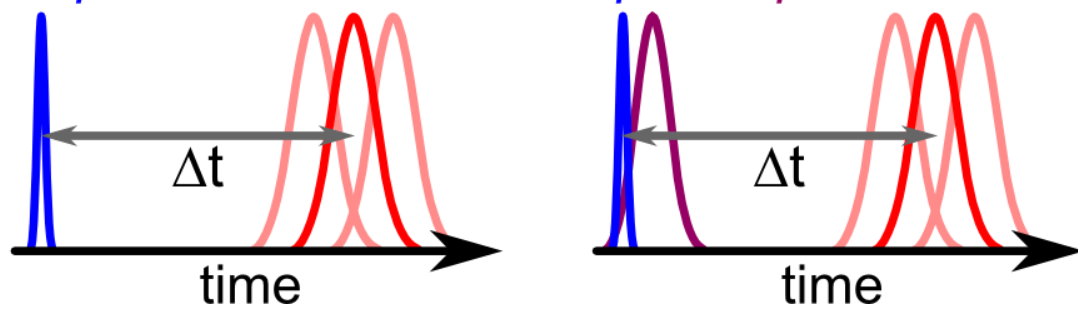
Figure 2
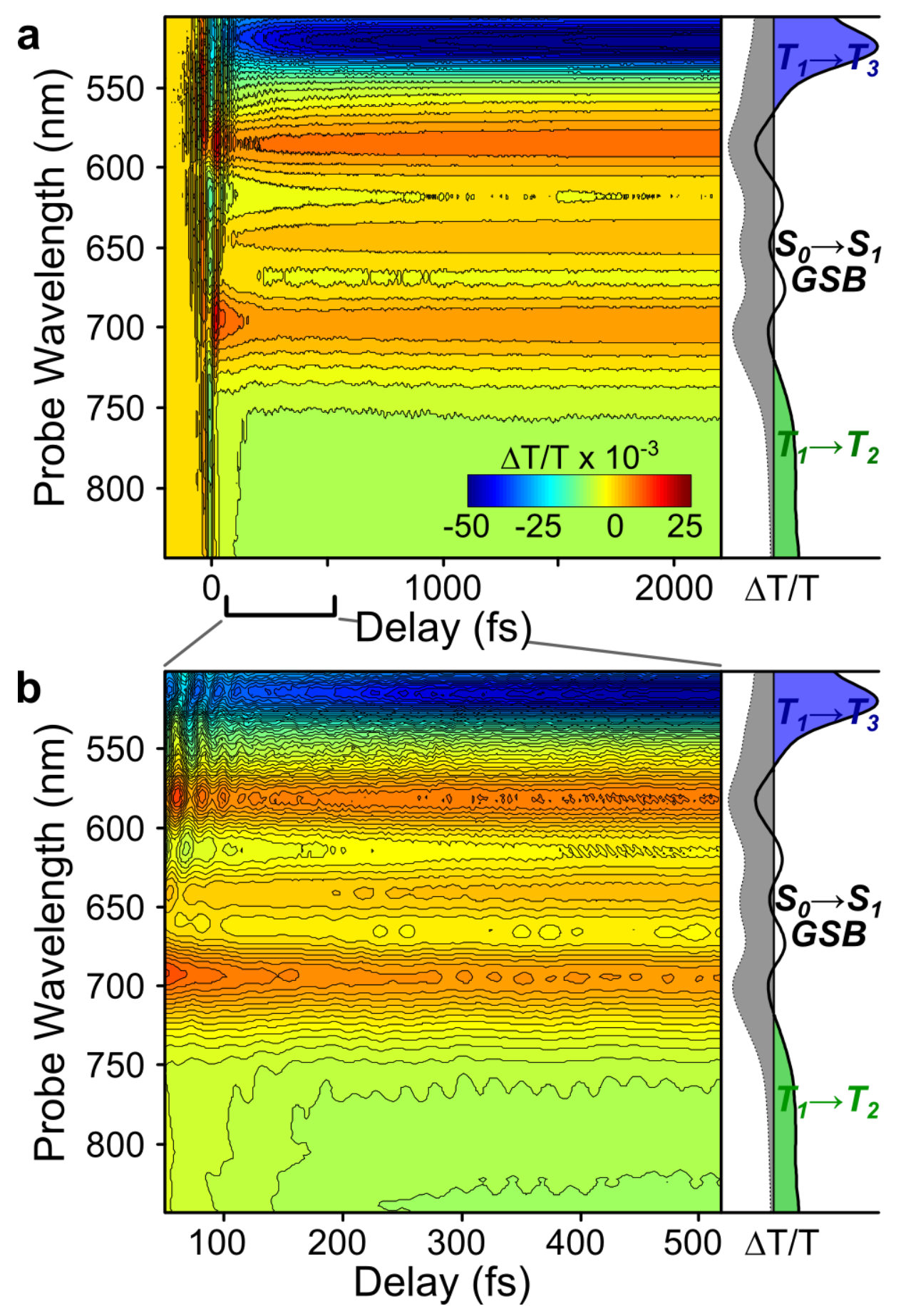


\section{Figure 3}
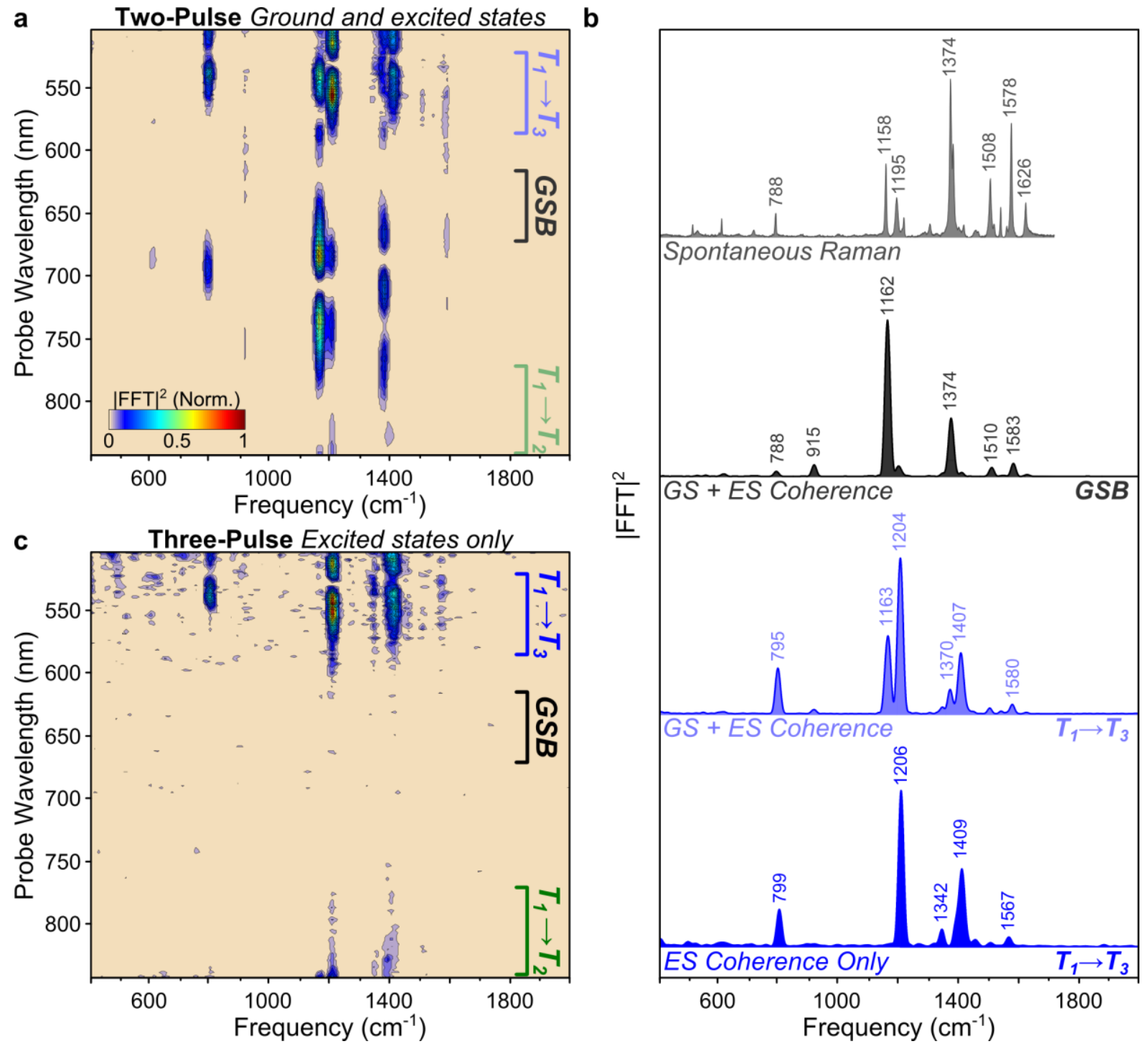
Figure 4

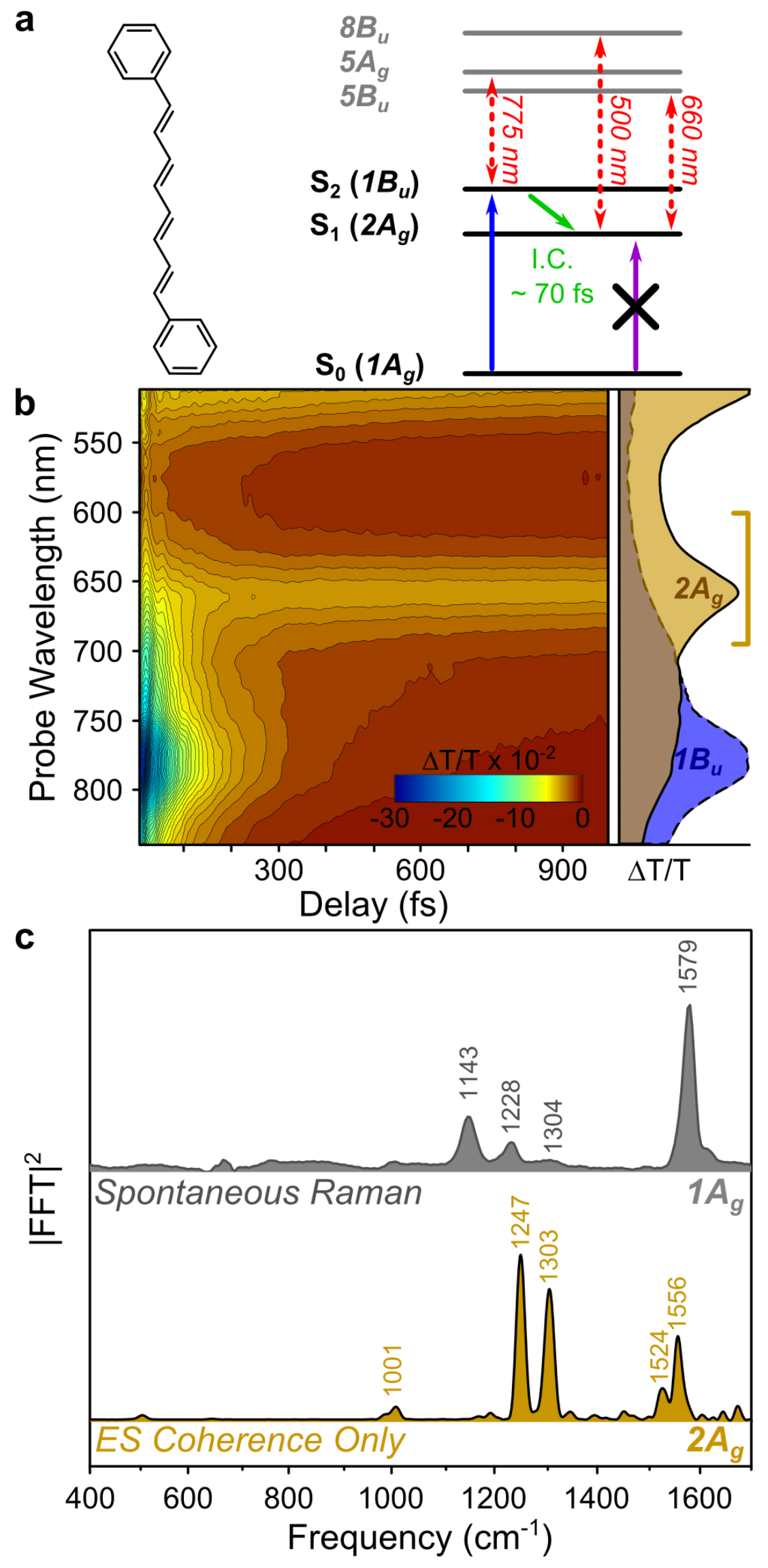


Figure 5

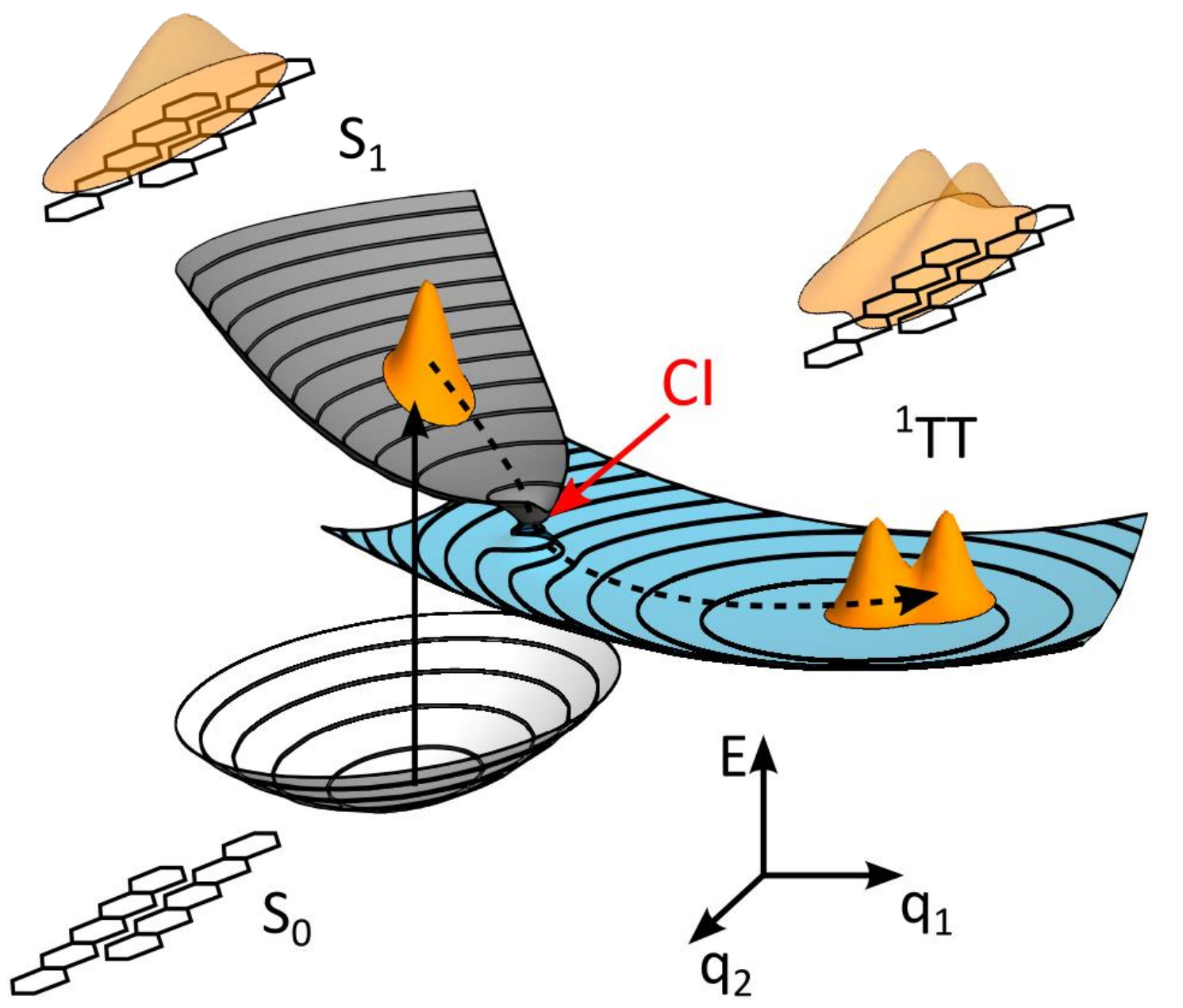

